\title{
Protistas ciliados e seu potencial uso como bioindicadores de qualidade de água
}

\author{
Ciliated protists and its potencial use as bioindicators of water quality
}

Protistas ciliados y su uso potencial como bioindicadores de la calidad del agua

Juliana Heloisa Pinê Américo-Pinheiro

Professora Doutora, Fundação Educacional de Andradina (FEA), Andradina - SP. E-mail: americo.ju@gmail.com

\author{
Nádia Hortense Torres \\ Pós-doutoranda, Laboratório de Tratamento de Resíduos e Efluentes, \\ Universidade Tiradentes (Unit), Aracajú - SE. E-mail: nadiahortense@gmail.com \\ Luiz Fernando Romanholo Ferreira \\ Professor Doutor, Universidade Tiradentes (Unit), \\ Aracajú - SE. E-mail: romanholobio@gmail.com
}

\begin{abstract}
RESUMO: A contaminação dos ecossistemas aquáticos por substâncias químicas provenientes de atividades agrícolas inadequadas e do despejo irregular de efluentes tem se intensificado. Assim, é indispensável o uso de organismos que possam indicar a presença desses contaminantes ou a qualidade dos corpos hídricos. $\mathrm{O}$ objetivo do presente trabalho foi realizar uma revisão de bibliográfica sobre os aspectos gerais dos protistas ciliados ressaltando a importância da utilização desses organismos como bioindicadores em estudos de monitoramento de qualidade de água e em ensaios de ecotoxicidade com contaminantes aquáticos. De acordo com essa pesquisa, os protistas ciliados são excelentes organismos para serem utilizados como bioindicadores de qualidade de água em programas de monitoramento devido à sua abundância, ciclo de vida curto, sensibilidade às alterações físicas, químicas e biológicas no ambiente aquático, facilidade de manutenção em laboratório e prevalência em corpos hídricos. Os estudos com esses ciliados podem ser focados tanto em análises da diversidade, riqueza e abundância de espécies em ecossistemas aquáticos como em avaliações da ecotoxicidade de contaminantes que podem atingir corpos d'água. Nos estudos de monitoramento da qualidade de água que incluem determinação da riqueza, abundância e diversidade em ambientes impactos as espécies identificadas podem variar de uma região para outra, enquanto que em ensaios laboratoriais de toxicidade as espécies dos gêneros Tetrahymena e Paramecium são internacionalmente as mais utilizadas.
\end{abstract}

PALAVRAS-CHAVE: Protozoários. Ecotoxicidade. Paramecium. Contaminantes.

ABSTRACT: Contamination of aquatic ecosystems by chemicals from inadequate agricultural activities and irregular effluent disposal has intensified. Thus, the use of organisms that may indicate the presence of these contaminants or the quality of the water bodies is indispensable. The objective of the present paper was to perform a literature review on the general aspects of ciliate protists emphasizing the importance of using these organisms as bioindicators in water quality monitoring studies and in aquatic contaminant ecotoxicity tests. According to this research, ciliary protists are excellent organisms to be used as bioindicators of water quality in monitoring programs due to their abundance, short life cycle, sensitivity to physical, chemical and biological changes in the aquatic environment, ease of maintenance In laboratory and prevalence in water bodies. Studies with these ciliates can be focused on analyzes of the diversity, richness and abundance of species in aquatic ecosystems as well as evaluations of the ecotoxicity of contaminants that can reach bodies of water. In water quality monitoring studies that include determination of richness, abundance and diversity in impacted environments the identified species may vary from one region to another, whereas in laboratory toxicity tests the species of the genera Tetrahymena and Paramecium are internationally most used . 
KEYWORDS: Protozoa. Ecotoxicity. Paramecium. Contaminants

RESUMEN: La contaminación de los ecosistemas acuáticos por los productos químicos provenientes de las actividades agrícolas inadecuadas y la descarga irregular de efluentes se ha intensificado. Por lo tanto, el uso de organismos que pueden indicar la presencia de estos contaminantes o la calidad de los cuerpos de agua es indispensable. El objetivo de este estudio fue realizar una revisión de la literatura sobre los aspectos generales de los protistas ciliados que enfatizan la importancia de usar estos organismos como indicadores biológicos en estudios de monitoreo de calidad del agua y las pruebas de ecotoxicidad con los contaminantes acuáticos. De acuerdo con esta encuesta, los protistas ciliados son excelentes organismos para ser usados como bioindicadores de la calidad del agua en los programas de vigilancia debido a su abundancia, corto ciclo de vida, sensibilidad a los cambios biológicos en el medio ambiente acuático física, química y facilidad de mantenimiento, laboratorio y prevalencia en los cuerpos de agua. Los estudios con estos ciliados pueden centrarse tanto en el análisis de la diversidad, riqueza y abundancia de las especies en los ecosistemas acuáticos como las evaluaciones de la ecotoxicidad de los contaminantes que pueden llegar a los cuerpos de agua. En los estudios de vigilancia de la calidad del agua, incluyendo la determinación de la riqueza, abundancia y diversidad de ambientes impactos especies identificadas pueden variar de una región a otra, mientras que en el laboratorio de pruebas de toxicidad de especies de géneros Tetrahymena y Paramecium son los más utilizados a nivel internacional .

PALABRAS-CLAVE: Protozoos. Ecotoxicidad. Paramecium. Contaminantes.

\section{INTRODUÇÃO}

A expansão dos grandes centros urbanos e industriais tem provocado uma progressiva degradação da qualidade dos recursos hídricos, devido ao aumento da contaminação de rios, lagos e reservatórios (AMÉRICO et al., 2012) por substâncias químicas provenientes de atividades agrícolas inadequadas e do despejo irregular de efluentes.

Como consequência da imensa quantidade e diversidade de substâncias que atingem os corpos d'água é indispensável o uso de metodologias adequadas que permitam a avaliação da qualidade da água. As metodologias tradicionais de avaliação, baseadas em características físicas, químicas e bacteriológicas, não são suficientes para atender aos usos múltiplos da água, sendo particularmente deficientes na avaliação da qualidade estética, de recreação e ecológica do ambiente (BUSS; BAPTISTA; NESSIMIAN, 2003).

Nesse sentido, ressalta-se a importância da utilização de organismos bioindicadores na avaliação da qualidade da água. Segundo Washington (1994), bioindicadores são espécies escolhidas por sua sensibilidade ou tolerância a vários parâmetros, como poluição orgânica ou outros tipos de poluentes.

Os organismos considerados como bioindicadores podem ser cultivados em laboratórios e utilizados para determinar a toxicidade de substâncias químicas puras, de misturas ou de efluentes sendo nesses casos conhecidos como organismos teste. De acordo com Rand e Petrocelli (1985), os critérios de seleção de organismos teste são: abundância e disponibilidade; significativa representação ecológica dentro das biocenoses; cosmopolitismo da espécie; profundo conhecimento da biologia, fisiologia e hábitos alimentares; estabilidade genética e uniformidade de suas populações; baixo índice de sazonalidade; sensibilidade constante e apurada; importância comercial; facilidade de cultivo em laboratório e, se possível, a espécie deve ser nativa para a melhor representatividade dos ecossistemas.

A importância da utilização de organismos da biota aquática como bioindicadores de contaminação ambiental é evidenciada em estudos com algas (FERREIRA et al., 2007), macrófitas aquáticas (SILVA et al., 2016), microcrustáceos 
(HAYASAKA et al., 2012; Ql et al., 2013 ), protistas (MADONI, 2000; LÁNG; KOHIDAl, 2012) e peixes (WANG et al., 2015).

Os protistas são organismos eucariotos pertencentes a comunidades planctônicas e bentônicas de ecossistemas aquáticos, nos quais desempenham papéis críticos tanto qualitativamente quanto quantitativamente (FENCHEL, 1987). Por serem organismos muito sensíveis a mudanças ambientais, os protistas são ideais como bioindicadores de alerta precoce da deterioração de ambientes aquáticos e como organismos teste para ensaios ecotoxicológicos (BERNARDO; BARP; STOLBERG. 2009; WU et al., 2015).

Entre os protistas indicadores de poluição de rios e riachos, temos os ciliados (Ciliophora), que devido a suas características de alta sensibilidade a pequenas variações no ambiente em que se encontram, são considerados excelentes bioindicadores (MADONI; ZANGROSSI, 2005). Ainda, devido ao fato de apresentarem ciclo de vida curto, permitem verificar os impactos em pequena escala de tempo, pois respondem diretamente às alterações no ambiente (CASTRO; BAPTISTA; BARRAUD, 2009).

O objetivo do presente trabalho foi realizar uma revisão de bibliográfica sobre os aspectos gerais dos protistas ciliados ressaltando a importância da utilização desses organismos como bioindicadores em estudos de monitoramento de qualidade de água e em ensaios de ecotoxicidade com contaminantes aquáticos.

\section{ASPECTOS GERAIS DOS PROTISTAS CILIADOS}

Os protistas incluem os eucariotos que não apresentam nível de organização tecidual observado nos Reinos Plantae, Fungi e Animalia (EVERT; EICHHORN, 2014). Os planos corpóreos dos protistas demonstram uma diversidade notável de forma, função e estratégias de sobrevivência. A maioria, mas não todos, é unicelular (BRUSCA; BRUSCA, 2007).

Por apresentarem grande superfície relativa e elevada taxa metabólica por unidade de peso, além de utilizarem várias formas de alimentação, os protistas são eficientes na absorção e liberação de nutrientes facilitando as trocas com o meio no qual estão inseridos. As altas taxas de crescimento e a capacidade de encistamento, especialmente no táxon Ciliophora, permitem que esses organismos apresentem maior eficiência na ocupação de nichos ecológicos (ARANTES JUNIOR et al., 2004). Segundo Sherr e Sherr (1988), os protistas são importantes elos nas cadeias tróficas aquáticas, ondem mediam o fluxo de substâncias e energia de um nível trófico para outro.

O táxon Ciliophora possui cerca de 12.000 espécies descritas (BRUSCA; BRUSCA, 2007), é constituído por organismos heterotróficos, exibindo uma ampla variedade de comportamentos alimentares e ampla distribuição geográfica (LYNN, 2008). As espécies desse táxon se locomovem por meio de cílios que são estruturas em forma de filamentos muito curtos distribuídos geralmente por quase todo corpo realizando batimentos coordenados (BRANCO, 1986). Os cílios também podem estar associados ao citóstoma e a área alimentar da célula protista auxiliando na alimentação. São organismos comuns nas comunidades bentônicas e planctônicas nos hábitats marinhos, de água salobra e de água doce, assim como nos solos úmidos (BRUSCA; BRUSCA, 2007). 
Os ciliados possuem dois tipos de núcleos, o macronúcleo e o micronúcleo. O macronúcleo possui a função de regular o metabolismo do organismo, a perda deste é considerada letal para o organismo (RAIKOV, 1996). O micronúcleo tem por função as atividades genéticas, podendo variar em tamanho de 1,5 e $5 \mu \mathrm{m}$ e podem apresentar formato esférico ou ovóide (CORLISS, 1979; LYNN; SMALL, 2002).

A reprodução assexuada nos ciliados, que ocorre por fissão binária é um processo que resulta em duas células-filhas semelhantes. Enquanto que na reprodução sexuada, por conjugação, ocorre a reversibilidade da união entre os conjugantes e a substituição do macronúcleo, sendo esta uma característica exclusiva dos ciliados (GRELL, 1973; CORLISS, 1979).

Alguns ciliados possuem pedúnculos contrácteis, podendo reunir-se em colônias como Vorticella e Epistylis (BRANCO, 1986), mas a maioria ocorre como células isoladas como Paramecium, Euplotes e Nassula (BRUSCA; BRUSCA, 2007).

Considerados como os organismos unicelulares mais complexos existentes, o ciliados apresentam características autoecológicas que são determinantes no processo de ciclagem e regulação ambiental (ARANTES JUNIOR et al., 2004). Estudos em lagos eutróficos indicam que a contribuição os ciliados é de $60 \%$ da biomassa total do zooplâncton (PACE; ORCUTT, 1981).

Protistas ciliados, tais como Tetrahymena e Colpidium são frequentemente utilizados como organismos modelos de experimentos para avaliar os efeitos de produtos químicos em protistas. Outras espécies são amplamente utilizadas como indicadores de qualidade de água e têm sido utilizados para clarificar água em estações de tratamento de água (BRUSCA; BRUSCA, 2007).

\section{UTILIZAÇÃo DE CILIADOS NO MONITORAMENTO DE QUALIDADE DE ÁGUA}

O monitoramento das variáveis físicas e químicas proporciona medida instantânea da qualidade da água, no entanto, a análise de variáveis biológicas permite a deteç̧ão e a integração das interferências que o ambiente sofre continuamente ou intermitentemente. $\mathrm{O}$ biomonitoramento utiliza como ferramentas fundamentais diversos índices ecológicos a fim de avaliar o grau de impacto nos ambientes aquáticos (SALUSSO; MORAÑA, 2002).

Os métodos que são baseados nas respostas de comunidade biológicas são considerados como as melhores formas de monitoramento e de avaliação da qualidade dos recursos hídricos, pois analisam seres vivos que dependem das condições ambientais e apresentam sensibilidade a contaminantes (MARQUES; BARBOSA, 2001).

Protistas ciliados são considerados como bioindicadores de qualidade da água, devido à sensibilidade desses organismos às alterações ambientais, às altas taxas de reprodução e variedade de nichos tróficos que ocupam, além de permitirem o monitoramento da qualidade da água em curtos períodos de tempo devido a sua elevada taxa metabólica (SALUSSO; MOREÑA, 2002; JIANG; SHEN, 2005; MADONI, 2005).

De acordo com Bernardo, Barp e Stolberg (2009), os protistas ciliados podem ser utilizados com bioindicadores em estudo de monitoramento de qualidade de água. Os autores ressaltam a importância das avaliações taxonômicas dos 
gêneros de ciliados (diversidade, abundância e riqueza) apontando indicações para a validade do uso dos organismos planctônicos para caracterização de impactos ambientais.

Espirito Santo (2012) avaliou a influência da poluição orgânica sobre a composição e estrutura da comunidade de protozoários ciliados em um sistema lótico no município de Juiz de Fora, Minas Gerais. A elevada dominância e baixa riqueza de ciliados caracterizaram córregos impactados (eutróficos), sendo que a espécie Carchesium polypinum ocorreu com maior frequência e abundância nos ambientes com altos níveis de poluição orgânica.

Em um estudo realizado na Represa das Antas - MG que é um ambiente com condições de oligotrofia foram registrados predomínio de ciliados pequenos com reduzido tempo de geração (Strombidium sp. e Enchelys sp.) e grandes ciliados menos vulneráveis a predação com Campanella umbellaria e Paradileptus elephantinus (RONQUI, 2008). O autor do estudo também salienta que esses gêneros encontrados na represa podem ser indicativos de condições de estresse devido à presença de agente químicos.

Tetrahymena pyriformis é um ciliado de água doce não patogênico cuja abundância pode indicar ambientes aquáticos saudáveis. Representa um nível trófico importante onde os processos de bioacumulação podem ser significativos (GERHARDT; UD-DAULA; SCHRAMM, 2010). Os gêneros Epistylis, Paramecium e Vorticella são associados à microfauna aquática de ambientes com carga orgânica moderada (LOPEZ; SAMPAIO, 2003).

Segundo Arantes Junior et al. (2004), os ciliados dos gêneros Epistylis e Vorticella são comuns em ambientes mesotróficos e eutróficos e normalmente estão associados ao fitoplâncton, que é utilizado como substrato de fixação. No estudo dos autores no Reservatório de Salto Grande, Americana - SP, o maior número de táxons dos ciliados foi encontrado na porção do reservatório menos poluída por resíduos industriais.

A população de ciliados é altamente exigente em relação ao hábitat, onde os impactos ambientais restringem a quantidade de gêneros no ambiente, possibilitando a proliferação descontrolada de indivíduos (BERNARDO; BARP; STOLBERG, 2009). Estudos indicam que quanto maior a diversidade de protozoários, melhor a qualidade da água (XU et al., 2008; JIANG et al., 2011).

\section{ECOTOXICIDADE DE CONTAMINANTES AQUÁTICOS PARA CILIADOS}

Diante da frequente contaminação dos ambientes aquáticos por compostos xenobióticos, a ecotoxicologia é uma ferramenta indispensável na avaliação da toxicidade desses contaminantes a organismos aquáticos expostos. Segundo Zagatto e Bertoletti (2006), por meio do uso de organismos teste é possível identificar compostos que afetam os sistemas biológicos, permitindo a avaliação do potencial risco ambiental de determinados contaminantes.

Os protozoários ciliados têm sido muito utilizados em estudos ecotoxicológicos devido à sua alta sensibilidade a xenobióticos, curto ciclo de vida e facilidade de cultivos e manutenção em laboratório (LÁNG; KOHIDAl, 2012; MIRANDA; MARTINS, 2013; WU et al., 2015). A prevalência dos ciliados em ecossistemas aquáticos também os torna organismos notáveis para avaliação da toxicidade de contaminantes aquáticos (ARANTES JUNIOR et al., 2004; RONQUI, 2008). 
A comunidade aquática de ciliado é constituída por um conjunto complexo de organismos que interagem, muitas vezes incluindo espécies sensíveis, resistentes ou com tolerância intermediária aos xenobióticos (MADONI, 2000). Destarte, o estudo da sensibilidade de protistas ciliados, a vários tipos de contaminantes, pode fornecer um padrão de medida para identificar a intensidade e o potencial dano ecológicos causado pelo aporte de substâncias tóxicas nos recursos hídricos (MADONI; ROMEO, 2006).

Os ciliados mais utilizados em ensaios de toxicidade são principalmente os do gênero Tetrahymena (GERHARDT; UDDAULA; SCHRAMM, 2010; LÁNG; KOHIDAI, 2012) e do gênero Paramecium (MIRANDA; MARTINS, 2013; WU et al., 2015), no entanto ensaios laboratoriais com outros gêneros de ciliados como Oxytrica fallax foram realizados com o objetivo de verificar a toxicidade de contaminantes emergentes para esses organismos (MADONI; ROMEO, 2006; AMANCHIE; HUSSAIN, 2010 ).

Ensaios de toxicidade do clorobenzeno para Tetrahymena indicaram que uma exposição em longo prazo a essa substância podem ser prejudiciais ao protista. O clorobenzeno pode ser absorvido e metabolizado pelo ciliado. Além disso, a exposição ao contaminante pode inibir o crescimento de Tetrahymena (ZHANG et al., 2012).

Os efeitos tóxicos dos antimicrobianos triclosan e triclocarban também foram observados em Tetrahymena thermophila. Os dois agentes tóxicos podem inibir o crescimento do protozoário em um período de 24 horas de exposição, sendo a CL50 24h do triclosan de 1,062 $\mathrm{mg} \mathrm{L}^{-1}$ e a CL50 24h do triclocarban de 0,295 $\mathrm{mg} \mathrm{L}^{-1}$ (GAO et al., 2015).

A ecotoxicidade dos agrotóxicos diuron e carbofuran para Paramecium caudatum foi analisada em ensaios de toxicidade aguda e crônica. O diuron foi mais tóxico para o protozoário (CL50 6h: 64,6 mg L ${ }^{-1}$ ) do que o carbofuran (CL50 6h: $142 \mathrm{mg} \mathrm{L}^{-1}$ ). Os ensaios crônicos mostraram que esses pesticidas causam diminuição significativa no crescimento populacional, no número de geração e na biomassa do $P$. caudatum (MANSANO et al., 2016).

Miranda e Martins (2013) avaliaram a toxicidade de metais para Paramecium caudatum, os resultados dos autores indicaram que o ciliado foi mais sensível ao mercúrio e ao cobre ( $C$ L50 24h: 0,021 $\mathrm{mg} \mathrm{L}^{-1}$ ) e menos sensível ao molibdênio (CL50 24h: 4,2 $\mathrm{mg} \mathrm{L}^{-1}$ ) e lítio (CL50 24h: 5,5 $\mathrm{mg} \mathrm{L}^{-1}$ ).

Em ensaios de toxicidade com níquel e 12 espécies de ciliados, Spirostomum teres e Paramecium bursaria apresentaram alta sensibilidade ao metal com CL50 $24 \mathrm{~h}$ de $0,17 \mathrm{mg} \mathrm{L}^{-1}$ e $0,36 \mathrm{mg} \mathrm{L}^{-1}$, respectivamente, sendo que Euplotes patella foi a espécie mais tolerante com $\mathrm{CL} 5024 \mathrm{~h}$ de $7,7 \mathrm{mg} \mathrm{L}^{-1}$. Assim, S. teres pode ser considerado um excelente bioindicador para avaliação de toxicidade de água contaminada com metais pesados (MADONI, 2000).

Madoni e Romeo (2006) verificaram a toxicidade aguda de 5 metais pesados para 4 espécies de ciliados de água doce (Colpidium colpoda, Dexiotricha granulosa, Euplotes aediculatus e Halteria grandinella). Os autores constataram diferenças na sensibilidade das 4 espécies, sendo que $H$. grandinella foi a mais sensível ao cádmio (CL50 24h: 0,07 mg $\mathrm{L}^{-1}$ ) e ao chumbo ( $\mathrm{CL50} 24 \mathrm{~h}: 0,12 \mathrm{mg} \mathrm{L}^{-1}$ ), enquanto que $E$. aediculatus foi mais sensível ao níquel $\left(0,03 \mathrm{mg} \mathrm{L}^{-1}\right)$. 


\section{CONSIDERAÇõES FINAIS}

Os protistas ciliados são excelentes organismos para serem utilizados como bioindicadores de qualidade de água em programas de monitoramento devido à sua abundância, ciclo de vida curto, sensibilidade às alterações físicas, químicas e biológicas no ambiente aquático, facilidade de manutenção em laboratório e prevalência em corpos hídricos. Os estudos com esses ciliados podem ser focados tanto em análises da diversidade, riqueza e abundância de espécies em ecossistemas aquáticos como em avaliações da ecotoxicidade de contaminantes que podem atingir corpos d'água.

Nos estudos de monitoramento da qualidade de água que incluem determinação da riqueza, abundância e diversidade em ambientes impactos as espécies identificadas podem variar de uma região para outra, enquanto que em ensaios laboratoriais de toxicidade as espécies dos gêneros Tetrahymena e Paramecium são internacionalmente as mais utilizadas.

\section{REFERÊNCIAS}

AMANCHI, R. N; HUSSAIN, M. M. Cytotoxicity assessment of monocrotophos in Paramecium caudatum and Oxytricha fallax. Journal of Environmental Biology, v.31, n.5, p.603-607, 2010.

AMÉRICO, J. H. P. et al. Desreguladores endócrinos no ambiente e seus efeitos na biota e saúde humana. Pesticidas, v. 22, p. 17-34, 2012.

ARANTES JUNIOR et al. Caracterização das populações de protozoários (Ciliophora e Rhizopoda) no Reservatório de Salto Grande, Americana, SP. In: ESPÍNDOLA, E. L. G.; LEITE, M. A.; DORNFELD, C. B. (Ed.). Reservatório de Salto Grande (Americana, SP): caracterização, impactos e propostas de manejo. São Carlos: RiMa, 2004. p. 155-177.

BERNARDO, E. L.; BARP, E.; STOLBERG, J. Caracterização de ciliados planctônicos do Rio Queimados, Concórdia - SC. Ágora: revista de divulgação científica, v.16, n.2, p. 504-511, 2009.

BRANCO, S. M. Hirobiologia aplicada à engenharia sanitária. 3ạ. ed. São Paulo: Cetesb/Ascetesb, 1986. 640p.

BRUSCA, R. C.; BRUSCA, G. J. Invertebrados. 2ạ. ed. Rio de Janeiro: Guanabara Koogan, 2007. 968p.

BUSS, D. F.; BAPTISTA, D. F.; NESSIMIAN, J. L. Bases conceituais para a aplicação de biomonitoramento em programas de avaliação da qualidade da água de rios. Caderno de Saúde Pública, v. 19, n. 2, p. 465-473, 2003.

CASTRO, L. M. A.; BAPTISTA, M. B.; BARRAUD, S. Proposição de metodologia para a avaliação dos efeitos da urbanização nos corpos de água. Revista Brasileira de Recursos Hídricos, v.14, p. 113-123, 2009.

CORLISS, J. O. The ciliated protozoa. London: Pergamon Press, 1979. 455p.

ESPIRITO SANTO, B. S. Composição e estrutura da taxocenose de ciliados peritríqueos (Ciliophora, Peritrichia) em ambientes lóticos com gradiente de poluição orgânica e aspectos ecológicos da relação epibiótica dde peritríqueos e moluscos gastrópodes. 2012. 94p. Dissertação (Mestrado em Comportamento e Biologia Animal) - Universidade Federal de Juiz de Fora, Juiz de Fora, 2012. 
EVERT, R. F.; EICHHORN, S. E. Raven - Biologia Vegetal. 8a ed. Rio de Janeiro: Guanabara Koogan, 2014. 856p.

FENCHEL, R. T. Ecology of protozoa - the biology of free-living phagotrophic protists. Journal of Basic Microbiology, v. 28, n. 9-10, p. $612-613,1987$.

FERREIRA, C.S.C. et al. Acute toxicity of oxytetracycline and florfenicol to the microalgae Tetraselmis chuii and to the crustacean Artemia parthenogenetica. Ecotoxicology and Environmental Safety, v.67, p.452-458, 2007.

GAO, L. et al. Effects of triclosan and triclocarban on the growth inhibition, cell viability, genotoxicity and multixenobiotic resistance responses of Tetrahymena thermophila. Chemosphere, v. 139, p. 434-440, 2015.

GERHARDT, A.; UD-DAULA, A.; SCHRAMM, K. W. Tetrahymena spp. (Protista, Ciliophora) as test species in rapid multilevel ecotoxicity tests. Anglais, 49, p. 271- 280, 2010.

GRELL, K.G. Protozoology. New York: Springer, 1973. 554p.

HAYASAKA, D. et al. Differences in susceptibility of five cladoceran species to two systemic insecticides, imidacloprid and fripronil. Ecotoxicology, v. 21, p. 421-427, 2012.

JIANG, J. G.; SHEN, Y. F. Use of the aquatic protozoa to formulate a community biotic index for na urban water system. Science of the Total Environment, v. 346, p. 99-111, 2005.

JIANG, Y., XU, H.; AL-RASHEID, K. A. S.; WARREN, A.; HU, X. Planktonic ciliate communities in a semi- enclosed bay of Yellow Sea, northern China: annual cycle. Journal of the Marine Biological Association of the United Kingdom, $v, 91$, n. 1, p. 97-105, 2011.

LÁNG, J.; KOHIDAI, L. Effects of the aquatic contaminant human pharmaceuticals and their mistures on the proliferation and migratory responses oh the bioindicator freshwater ciliate Tetrahymena. Chemosphere, v. 89, p. 592-601, 2012.

LÓPEZ, C.M.; SAMPAIO, E.V. A comunidade zooplanctônica no reservatório de Três Marias e no trecho do São Francisco a jusante. p. 93-104. In: GODINHO, H. P.; GODINHO, A. L. Águas, peixes e pescadores das Minas Gerais. Belo Horizonte: Editora PUC Minas, 2003. 468p.

LYNN. D. H; SMALL, E. B. An illustred guide to the Protozoa. In: LEE, J. J.; BRADBURY, P. C.; LEEDALE, G .F. (Ed.). Phylum Ciliophora. Society of Protozoologists, Kansas: Lawrence. 2002. p.371-656.

LYNN, D. H. The Ciliated Protozoa. 3a ed. Guelph: Springer, 2008. 605p.

MADONI, P. The acute toxicity of nickel to freshwater ciliates. Environmental Polluiton, v. 109, p. 53-59, 2000.

MADONI, P. Ciliated protozoan communities and saprobic evalution of water quality in the hilly zone of some tributaries of the Po River (northen Italy). Hydrobiologia, v. 541, p. 55-69, 2005.

MADONI, P.; ZANGROSSI, S. Ciliated protozoa and saprobical evaluation of water quality in the Taro River (northern Italy). Italian Journal of Zoology, v. 72, p. 21-25, 2005.

MADONI, P.; ROMEO, M. G. Acute toxicity of heavy metals towards freshwater ciliated protists. Environmental Pollution, v. 141, p. 1-7, 2006.

MANSANO, A. S. et al. Effects of diuron and carbofuran pesticides in their purê and comercial forms on Paramecium caudatum: The use of protozoan in ecotoxicology. Environmental Pollution, v. 213, p. 160-172, 2016. 
MARQUES, M. M.; BARBOSA, F. Na fauna do fundo o retrato da degradação. Revista Ciência Hoje, v. 30, n. 175, p. 7275, 2001.

MIRANDA, M. M. P.; MARTINS, N. F. Testes de toxicidade de íons metais usando Paramecium caudatum como organismo teste. Revista Brasileira de Agrotecnologia, v. 3, n.1, p. 1-16, 2013.

PACE, M.L.; ORCUTT JR, J.D. The relative importance of protozoans, rotifers and crustaceans in a freshwater zooplankton community. Limnology and Oceanography, v. 36, p. 822-830, 1981.

QI, S. et al. Toxicity assessments with Daphnia magna of guadipyr, a new neonicotinóide insecticide and studies of its effect on acetylcholinesterase (AChE), glutathione S-transferase (GST), catalase (CAT) and chitobiase activities. Ecotoxicology and Environmental Safety, v.98, p. 339-344, 2013.

RAIKOV, I. B. Nuclei of ciliates. In: HAUSMANN, K.; BRADBURY (Ed). Ciliates. Cell as organisms. Stuttgart: Gustav Fisher Verlag, 1996. P. 221-242.

RAND, G. M.; PETROCELLI, S. R. Fundamentals of aquatic toxicology: methods and applications. Washington: Hemisphere, 1985. 665p.

RONQUI, L. B. Caracterização limnológica e avaliação de efeitos ambientais causados por efluentes de mina de urânio sobre populações microbianas planctônicas da Represa das Antas, Caldas (MG). 2008. 133p. Dissertação (Mestrado em Biotecnologia) - Universidade de São Paulo, São Paulo, 2008.

SALUSSO, M. M.; MORAÑA, L. B. Comparación de índices bióticos utilizados en el monitoreo de dos sistemas lóticos del noroeste argentino. Revista de Biologia Tropical, v.50, n.1, p. 327-336, 2002.

SHERR, E.; SHERR, B. Role of microbes in pelagic food webs: A revised concept. Limnology and Oceanography, v. 33, p. $1225-1227,1988$.

SILVA, A. F. et al. Sensitivity of aquatic organisms to ethanol and its potencial use as bioindicators. Acta Scientiarum Biological Sciences, v. 38, n. 4, p. 377-385, 2016.

XU, H.; SONG, W.; WARREN, A.; AL-RASHEID, K. A. S.; AL-FARRAJ. S. A.; GONG, J.; HU, X. Planktonic protist communities in a semi-enclosed mariculture pond: structural variation and correlation with environmental conditions. Journal of the Marine Biological Association of the United Kingdom, v. 88, n. 7, p. 1353-1362, 2008.

ZAGATTO, P. A.; BERTOLETTI, E. Ecotoxicologia. In: ZAGATTO, P. A. (Ed.). Ecotoxicologia aquática. São Carlos: RiMa, 2006. p. 1-12.

ZHANG, T. et al. Acute toxicity of chlorobenzenes in Tetrahymena: Estimated by microcalorimetry and mechanism. Environmental Toxicology and Pharmacology, v. 33, p. 377-386, 2012.

WANG, Y. et al. Assessing joint toxicity of four organophosphate and carbamato insecticides in common carp (Cyprinus carpio) using acetylcholinesterase activity as an endpoint. Pesticide Biochemistry and Physiology, v. 122, p. 81-85, 2015.

WASHINGTON, H. G. Diversity, biotic and similarity indices. A review with special relevance to aquatic ecosystems. Water Research, v. 18, p.653-694, 1984.

WU, D. et al. Inhibitory effect of three heavy mental pollutants on antioxidante enzymes of Paramecium caudatum. Advanced Materials Research, v. 1065, p. 3071-3076, 2015. 